\title{
Compact High-Power Tunable Three-Level Operation of Double Cladding Nd-Doped Fiber Laser
}

\author{
L. B. Fu, M. Ibsen, D. J. Richardson, J. Nilsson, D. N. Payne, and A. B. Grudinin
}

\begin{abstract}
We present a compact high-power continuous-wave tunable neodymium-doped double cladding fiber laser operating on three-level ${ }^{4} \mathbf{F}_{3 / 2}-{ }^{4} \mathbf{I}_{9 / 2}$ transition with a maximum output power up to $810 \mathrm{~mW}$. At $926.7 \mathrm{~nm}$, it has a maximum slope efficiency of $49.3 \%$ against absorbed $808-\mathrm{nm}$ pump. By compressing the fiber Bragg grating, 15-nm tuning range is achieved.
\end{abstract}

Index Terms-Double-cladding fiber laser, Nd fiber laser, threelevel transition, tunable laser.

\section{INTRODUCTION}

$\mathbf{T}$ HE OPERATION of three-level transition ${ }^{4} \mathrm{~F}_{3 / 2}-{ }^{4} \mathrm{I}_{9 / 2}$ $(890-950 \mathrm{~nm})$ of Nd-doped fiber laser is of great interest as it has many applications in water-vapor absorption characterization, frequency doubling, lidar, spectroscopy, etc. In these applications, narrow spectral width, high power, and tunable output is highly desirable. However, achieving high-power tunable laser output has long been a great challenge in three-level transition in Nd-doped fiber lasers. Similar to the well-known challenges in three-level transition operation of Yb-doped double cladding fiber lasers [1], operating three-level transition in Nd-doped double cladding fiber lasers also has great difficulty not only due to the existence of undesired extremely serious competition with four-level ${ }^{4} \mathrm{~F}_{3 / 2}{ }^{-4} \mathrm{I}_{11 / 2}$ transition when pumped at $808 \mathrm{~nm}$, but also due to the very poor overlap between pump and signal. Hence, laser operations of the ${ }^{4} \mathrm{~F}_{3 / 2}-{ }^{4} \mathrm{I}_{9 / 2}$ transition in Nd-doped optical fibers have been limited to low power.

Previously, laser operation power of Nd-doped fiber laser was reported to be $\sim 40 \mathrm{~mW}$ using a core pumping scheme [2]. Although under very low temperature (Nd-doped double cladding fibers cooled by liquid nitrogen), $2.1 \mathrm{~W}$ has been demonstrated using a master oscillation optical amplifier configuration [3]. Further, recently $11 \mathrm{~W}$ at $938 \mathrm{~nm}$ has been demonstrated in a follow-up experiment using a low numerical aperture (NA) fiber and higher pump power [4]; this has changed the 938-nm operation to a four-level transition under very low temperature, but such operations at low temperature are not desirable in many applications. Using a cladding-pumped amplifier which consists of Nd-doped fibers with a core structure of two concentric zones, Kane recently demonstrated a 3-W blue source from

Manuscript received August 9, 2004; revised October 2, 2004.

L. B. Fu was with ORC-University of Southampton, Southampton SO17 1BJ, U.K. He is now with CUDOS, School of Physics, University of Sydney, Sydney NSW 2006, Australia (e-mail: libin@ physics.usyd.edu.au).

M. Ibsen, D. J. Richardson, J. Nilsson, and D. N. Payne are with ORC-University of Southampton, Southampton SO17 1BJ, U.K.

A. B. Grudinin is with the Fianium-NewOptics Ltd., Southampton, U.K. (e-mail: abg@fianium.com).

Digital Object Identifier 10.1109/LPT.2004.840034

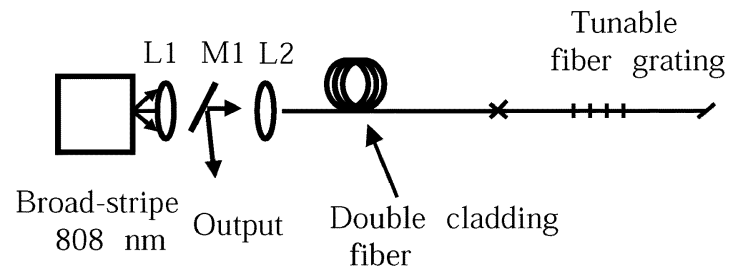

Fig. 1. Experimental setup (L1, L2: lens; M1: mirror).

a 914-nm Nd : $\mathrm{YVO}_{4}$ passively $Q$-switched laser [5]. Nevertheless, the highest output power of tunable laser ever reported is only $32 \mathrm{~mW}$ (from 896 to $939.5 \mathrm{~nm}$ ) using core pumping [6]. Most recently, although by combining two 808-nm pump diodes and bulk diffractive gratings, $2.4-\mathrm{W}$ peak power has been achieved, the laser is bulky, expensive, and far from compact [7]. In this letter, we report a very compact, highly stable, robust, and as high as $810-\mathrm{mW}$ output from a double cladding Nd-doped fiber laser with a tuning range of $15 \mathrm{~nm}$, which we confidently expect to be further extended. When operating at $926.7 \mathrm{~nm}$, the laser has a threshold of $1.3 \mathrm{~W}$ (launched pump power) and a slope efficiency of $17 \%$ against launched high-brightness pump at $808 \mathrm{~nm}$ (49.3\% against absorbed pump). In order to suppress the 1060-nm emission, we have designed the Nd-doped fiber with a W-type refractive index and doping profile [8]. In addition, the laser spectral width is very narrow (full-width at halfmaximum is about $0.11 \mathrm{~nm}$ ), a result that we attribute mainly to the compact, narrow-bandwidth, and tunable fiber Bragg grating as the high reflective mirror, which is based on the compression tunable fiber grating technique [9], [10].

\section{EXPERIMENTAL SETUP}

The experimental setup is shown in Fig. 1. Pump laser beam from a high brightness broad-stripe 808-nm pump diode is collimated using a lens L1 and then launched into Nd-doped double cladding fiber. The maximum pump power from the broad-stripe 808-nm diode is about 7.5 W. Mirror M1 has $98 \%$ transmission at pump wavelength and $99 \%$ reflectivity at $900-1100 \mathrm{~nm}$. The total launching efficiency is about $84 \%$ for the pump.

The fibers employed in this experiment are high concentration Nd-doped alumino-silicate fibers. To enhance the pump absorption efficiency, the inner cladding is designed to be a square shape with $\sim 100 \mu \mathrm{m}$ side coated with low-index polymer, giving a nominal NA to be 0.4 . The core radius is $5.5 \mu \mathrm{m}$ with an NA of 0.1 . More fabrication details about this Nd-doped fiber can be found in [11]. High Nd-doping enables high pump absorption (about $0.3 \mathrm{~dB} / \mathrm{m}$ ) and, thereby, short length can be used. Besides, 1060-nm band is greatly suppressed by W-type refractive index and doping profile. Since the signal at $1060 \mathrm{~nm}$ is more loosely confined than that at $930 \mathrm{~nm}$, 


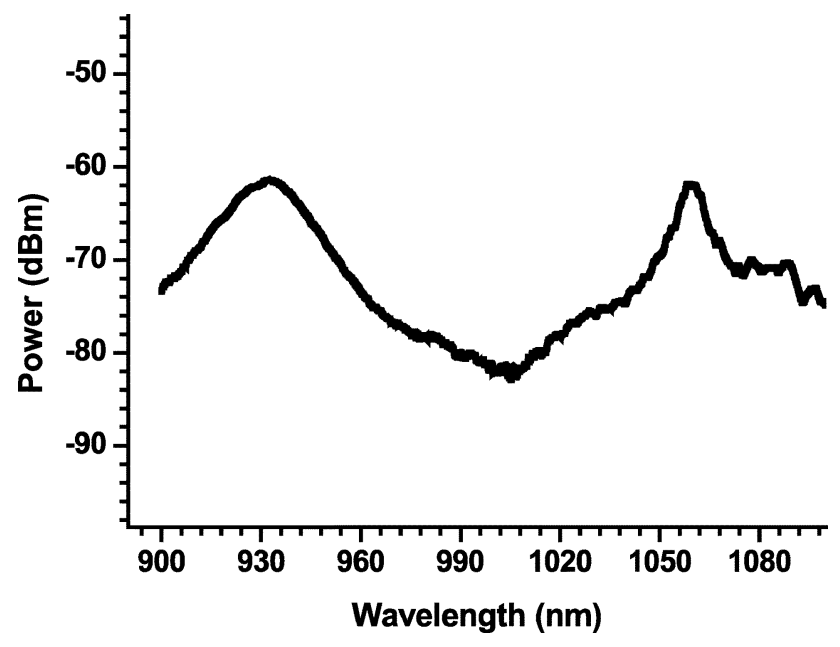

Fig. 2. Spontaneous emission from 6.1-m Nd-doped double cladding fiber.

the $\mathrm{Nd}$-doped fibers are coiled with a bending radius around $2.5 \mathrm{~cm}$ to further suppress the four-level ${ }^{4} \mathrm{~F}_{3 / 2}-{ }^{4} \mathrm{I}_{11 / 2}$ transition at $1.06 \mu \mathrm{m}$ when pumped at $808 \mathrm{~nm}$. When the Nd-doped fiber is pumped by $808-\mathrm{nm}$ high brightness broad-stripe diode, the spontaneous emission at $1060 \mathrm{~nm}$ is greatly suppressed, as is shown in Fig. 2. Note that due to the codoped aluminum, the peak of the fluorescence of this Nd-doped fiber is blue-shifted to be around $932 \mathrm{~nm}$ [12]. In three-level transition ${ }^{4} \mathrm{~F}_{3 / 2}-{ }^{4} \mathrm{I}_{9 / 2}$, there is a balance between signal reabsorption and pump utilization. Initially, we chose a Nd-doped fiber of $8 \mathrm{~m}$. Then, by cutting back some of the Nd-doped fibers and characterizing the maximal slope efficiency for each step, the optimal length is given to be around $6.1 \mathrm{~m}$ without utilizing pump feedback.

Thereafter, the fiber is spliced to a tunable fiber grating with an initial wavelength at $926.7 \mathrm{~nm}$ and the splicing loss is estimated to be around $1.4 \mathrm{~dB}$. The splice loss is high because the NA of this Nd-doped fiber is only 0.10 , while the photosensitive fiber used to write a fiber Bragg grating is 0.12 , leading to mode mismatch between the two fibers. However, if we design a photosensitive fiber with an NA of 0.10 , the splice loss $<0.1 \mathrm{~dB}$ can be easily achieved. Hence, much higher slope efficiency can be achieved.

The fiber Bragg grating is $1 \mathrm{~cm}$ long with a reflectivity of $98.3 \%$ at $926.7 \mathrm{~nm}(3-\mathrm{dB}$ full bandwidth $\sim 0.37 \mathrm{~nm})$. To form a cavity, the fiber at the pump end is flatly cleaved to make a mirror of $4 \%$ reflectivity. The compression tuning technique used in this experiment is the same as that used in [9] and [10]. The basic idea is that when a fiber Bragg grating is mounted on a beam, it will experience different strain relative to the neutral axis by varying bending radius, enabling compression tuning of the Bragg wavelength through elastic-optic effect.

\section{EXPERIMENTAL RESULTS AND DISCUSSION}

When the fiber Bragg grating is set at its original Bragg wavelength at $926.7 \mathrm{~nm}$, the laser has got maximal output with launched pump power of $6.3 \mathrm{~W}$. Fig. 3 demonstrates the optical spectrum when the laser is operating at this wavelength. The spectrum shows that the main desired signal at $926.7 \mathrm{~nm}$ is $17 \mathrm{~dB}$ higher than that at $1061 \mathrm{~nm}$. We estimate the bending loss to be around $0.5 \mathrm{~dB}$ for $1060 \mathrm{~nm}$ and $0.05 \mathrm{~dB}$ for $926 \mathrm{~nm}$. Because the high splicing loss point has reflection for

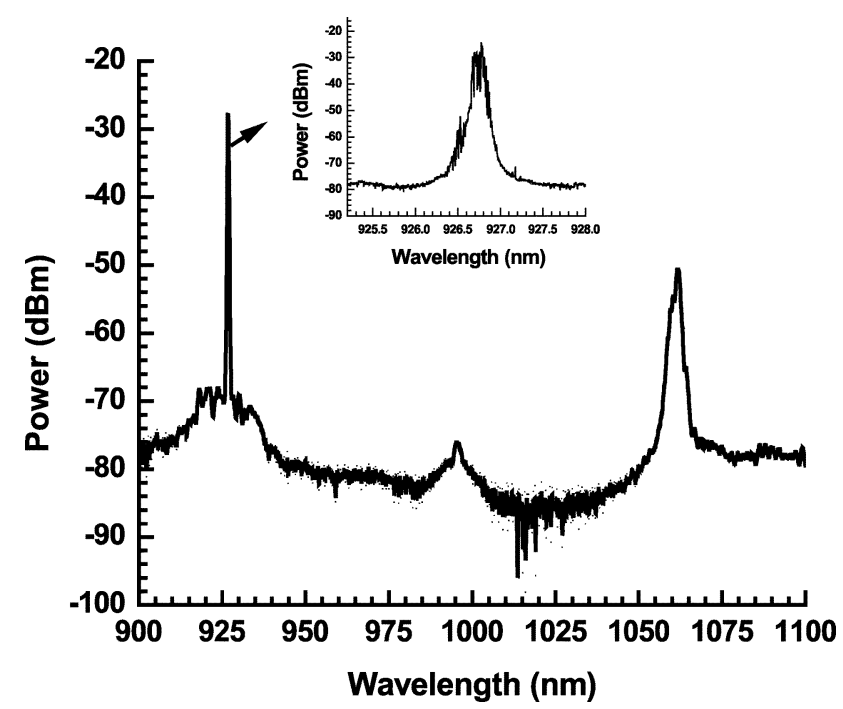

Fig. 3. Optical spectrum of the laser (resolution: $0.2 \mathrm{~nm}$ ), the inset is the spectrum around $926.7 \mathrm{~nm}$, (resolution: $0.05 \mathrm{~nm}$ ).

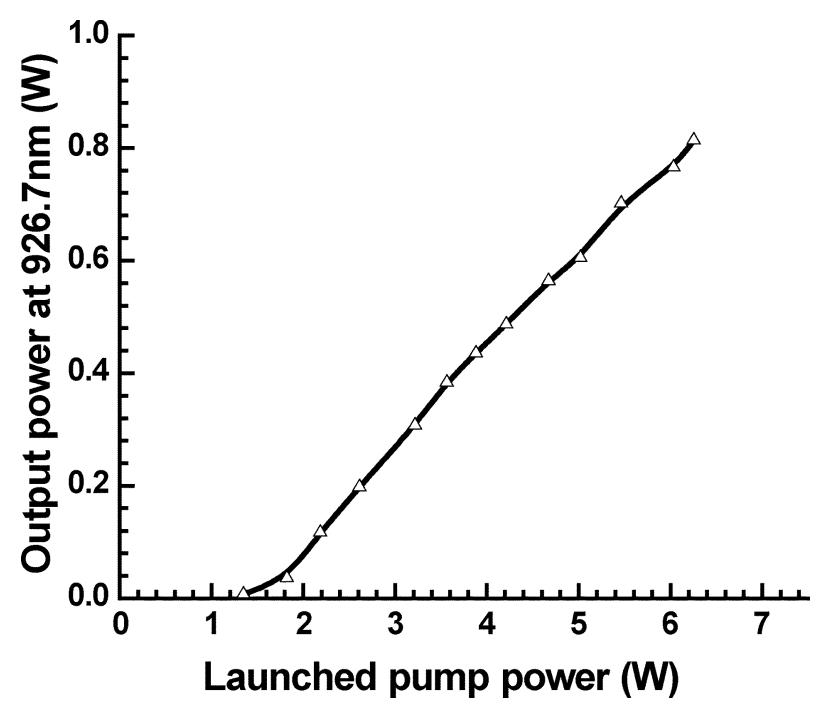

Fig. 4. Slope efficiency of the double cladding Nd-doped fiber laser at $926.7 \mathrm{~nm}$.

$1060 \mathrm{~nm}$ and it forms a cavity together with the other cleaved end, 1060-nm lasing still occurs at high pump power. When the pump power is lower, the 1061-nm signal disappears. If splicing is optimized, lasing at $1060 \mathrm{~nm}$ can be completely eliminated. Alternatively, we could bend the fibers with smaller radius, which however, would result in more pump loss. Hence, we keep a bending radius at $2.5 \mathrm{~cm}$. Meanwhile, the slope efficiency is characterized as is shown in Fig. 4. The lasing threshold is around $1.3 \mathrm{~W}$ (launched pump power). We attribute the high threshold to two factors. First, significant ground state absorption exists in this transition. Second, the mode mismatch between the $\mathrm{Nd}$-doped fiber and the single-mode fiber for grating writing leads to high splicing loss. The maximal slope efficiency is $17 \%$ against launched pump power while it is $49.3 \%$ against absorbed pump power. The power variations are below $1 \%$ of the average output power in $10 \mathrm{~min}$ when the laser is operating at the highest pump power of $6.3 \mathrm{~W}$. To investigate the output beam qualities, we have checked the $M^{2}$ of the 


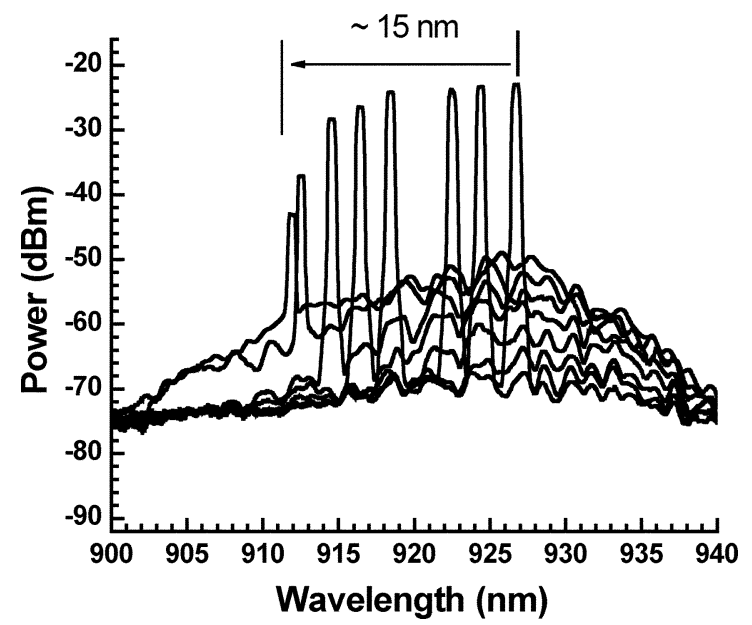

Fig. 5. Output spectra during tuning (RB: $0.5 \mathrm{~nm}$ ).

output beam to be 1.1 when the launched pump is around $6 \mathrm{~W}$. The laser output beam is indeed single spatial transverse mode.

Then we tuned the Bragg wavelength of the fiber grating to investigate the lasing properties of this laser at different wavelengths. Under the same pump power (launched power of $6 \mathrm{~W}$ ), the output is characterized as is depicted in Fig. 5. When the laser is tuned to $911.8 \mathrm{~nm}$, the amplified spontaneous emission begins to increase. If we optimize the splice between the Nd-doped fiber and the fiber Bragg grating, the cavity loss will be even lower and the laser can continue to work below $910 \mathrm{~nm}$ when the grating is further compressed. When the laser is tuned to $911.8 \mathrm{~nm}$, its threshold is around $4.6 \mathrm{~W}$ and its slope efficiency against launched power is $2.4 \%$.

The current tuning range has been limited mainly by the Bragg wavelength of the phase masks available to our laboratory. If the Bragg wavelength determined by the phase mask can be $950 \mathrm{~nm}$, we shall be able to easily tune the laser from 950 to $910 \mathrm{~nm}$ by using the afore-mentioned bending technique as the Bragg wavelength of a fiber Bragg grating can be tuned over 100 -nm range using a simple compression tuning technique [13].

We have also briefly investigated the power output of the double-clad fiber laser employing a bulk dichroic mirror as a complete reflective mirror both at pump and signal wavelength. In this case, there is no splice loss in the cavity. The Nd-doped double-clad fiber length is extended to $9.2 \mathrm{~m}$ to enable enough pump absorption to avoid any possible damage to the broadstripe pump diodes caused by reflected pump from the reflective mirror. The lasing threshold is around $1.42 \mathrm{~W}$ and the slope efficiency is $33.8 \%$ against launched pump power. The maximum power is around $1.55 \mathrm{~W}$ at a launched pump power of $6.3 \mathrm{~W}$ and the lasing longitudinal modes are between 920-932 nm.

If a better dichroic mirror is available, which allows high transmission at $800 \mathrm{~nm}$ but at the same time high reflection at $900-980 \mathrm{~nm}$ and low reflection at $1000-1100 \mathrm{~nm}$, we can easily change the output to a low reflection tunable fiber Bragg grating side. This configuration facilitates easy connection to high-power Nd-doped optical fiber amplifiers.

\section{CONCLUSION}

We have demonstrated a very compact, robust, ultrastable, continuous-wave tunable $\mathrm{Nd}$-doped double cladding fiber laser based on the transition ${ }^{4} \mathrm{~F}_{3 / 2}-{ }^{4} \mathrm{I}_{9 / 2}$ with output as high as $810 \mathrm{~mW}$. To overcome the very strong competition from four-level ${ }^{4} \mathrm{~F}_{3 / 2}-{ }^{4} \mathrm{I}_{11 / 2}$ transition, we employed a high brightness broad-stripe pump diode and designed the fiber with $\mathrm{W}$-type refractive index and doping profile plus bending loss to suppress 1060-nm band emission. The laser shows a maximum slope efficiency of $49.3 \%$ at $926.7 \mathrm{~nm}$ against absorbed pump power. In addition, it is operating in a single spatial mode. By compression-tuning the fiber Bragg grating, 15-nm tuning range is achieved. With such a simple, low-cost, compact, and highly reliable compression tuning technique, we confidently expect that a further extension of the tuning range to be as high as $40 \mathrm{~nm}$ can be implemented.

\section{REFERENCES}

[1] R. Selvas, J. K. Sahu, L. B. Fu, J. N. Jang, J. Nilsson, A. B. Grudinin, K. H. Ylä-Jarkko, S. A. Alam, P. W. Turner, and J. Moore, "High-power, low-noise, Yb-doped, cladding-pumped, three-level fiber sources at 980 nm," Opt. Lett., vol. 28, no. 13, pp. 1093-1095, Jul. 2003.

[2] P. Dragic and G. Papen, "Fiber amplification in the 940-nm water vapor absorption band using the ${ }^{4} \mathrm{~F}_{3 / 2}-{ }^{4} \mathrm{I}_{9 / 2}$ transition in Nd," IEEE Photon. Technol. Lett., vol. 9, no. 11, pp. 1478-1480, Nov. 1997.

[3] J. Dawson, R. Beach, A. Drobshoff, Z. Liao, D. Pennington, and S. Payne, "938 nm Nd-doped high power cladding pumped fiber amplifier," in 2003 Advanced Solid-State Photonics Tech. Dig., Santa Antonio, TX, Feb. 2-5, Paper MD3.

[4] J. W. Dawson, R. Beach, A. Drobshoff, Z. Liao, D. M. Pennington, S. A. Payne, L. Taylor, W. Hackenberg, and D. Bonaccini, "Scalable 11 W $938 \mathrm{~nm} \mathrm{Nd}{ }^{3+}$ doped fiber laser," presented at the Proc. Advanced Solid-State Photonics, Santa Fe, NM, Feb. 2-5, 2004, Paper MD8.

[5] T. J. Kane, G. Keaton, M. A. Arbore, D. R. Balsley, J. F. Black, J. L. Brooks, M. Byer, L. A. Eyres, M. Leonardo, J. J. Morehead, C. Rich, D. J. Richard, L. A. Smoliar, and Y. Zhou, "3-watt blue source based on 914-nm Nd:YVO4 passively- $Q$-switched laser amplified in claddingpumped Nd fiber," presented at the Proc. Advanced Solid-State Photonics 2004, Santa Fe, NM, Feb. 2-5, Paper MD7.

[6] A. L. Cook and H. D. Hendricks, "Diode-laser-pumped tunable 896-939.5-nm neodymium-doped fiber laser with 43-mW output power," Appl. Opt., vol. 37, no. 15, pp. 3276-3280, May 1998.

[7] D. B. S. Soh, S. W. Yoo, J. Nilsson, J. K. Sahu, S. Baek, Y. Jeong, L. J. Cooper, C. Codemard, P. Dupriez, C. Alegria, V. Philippov, and K. Oh, "Cladding pumped Nd-doped fiber laser tunable from $908 \mathrm{~nm}$ to 938 nm," in Conf. Lasers Electro-Optics/Int. Quantum Electronics Conf. (CLEO/IQEC) 2004, San Francisco, CA, Paper CMK4.

[8] I. V. Neves and A. S. C. Fermandes, "Modal characteristics for W-Type and M-Type dielectric profile fibers," Microw. Opt. Technol. Lett., vol. 22, no. 6, pp. 398-405, 1999.

[9] C. S. Goh, S. Y. Set, and K. Kikuchi, "Widely tunable optical filters based on fiber Bragg gratings," IEEE Photon. Technol. Lett., vol. 14, no. 9, pp. 1306-1308, Sep. 2002

[10] L. Fu, M. Ibsen, M. Mokhtar, M. Gunning, D. Richardson, and D. Payne, "Wideband compression-tuned all-fiber DFB laser: analysis and characterization," in OFC 2003, vol. 1, Atlanta, GA, Paper TUL3, pp. 235-236.

[11] I. A. Bufetov, V. V. Dudin, A. V. Shubin, A. K. Senatorov, E. M. Dianov, A. B. Grudinin, S. E. Goncharov, I. D. Zalevskii, A. N. Gur'yanov, M. V. Yashkov, A. A. Umnikov, and N. N. Vechkanov, "Efficient 0.9-mm neodymium-doped single-mode fiber laser," Quantum Electron., vol. 33, no. 12, pp. 1035-1037, 2003.

[12] K. Arai, H. Namikawa, K. Kumata, and T. Honda, "Aluminum or phosphorous co-doping effects on the fluorescence and structural properties of neodymium-doped silica glass," J. Appl. Phys., vol. 59, pp. 3430-3436, 1986.

[13] M. R. Mokhtar, C. S. Goh, S. A. Butler, S. Y. Set, K. Kikuchi, D. J. Richardson, and M. Ibsen, "Fiber Bragg gratings compression-tuned over 110 nm," Electron. Lett., vol. 39, no. 6, pp. 509-511, 2003. 